\title{
The Hydration of a DNA-Amphiphile Complex
}

\author{
Cecilia Leal, ${ }^{* \dagger} \nmid$ Lars Wadsö, ${ }^{\dagger}$ Gerd Olofsson, ${ }^{\dagger}$ Maria Miguel, ${ }^{\S}$ and Håkan Wennerström ${ }^{\dagger}$ \\ Physical Chemistry 1, Lund University, POB 124, 22100 Lund, Sweden, Building Materials, Lund University, \\ POB 118, 22100 Lund, Sweden, and Chemistry Department, University of Coimbra, \\ 3004-535 Coimbra, Portugal
}

Received: July 15, 2003; In Final Form: December 11, 2003

\begin{abstract}
We present measurements of isothermal DNA-hexadecyltrimethylammonium (DNACTA) complex and pure DNA hydration at $25{ }^{\circ} \mathrm{C}$ using a sorption microcalorimeter. This calorimeter provides simultaneous measurement of (i) water activity (sorption isotherms) and (ii) the partial molar enthalpy of water as a function of water uptake. For pure DNA, hydration is exothermic over the studied concentration range and we find an approximately linear relation between the partial molar enthalpy and the partial molar free energy. A kink in the isotherm appears at $20.0 \pm 0.3$ water molecules per base pair for a water activity of 0.80 , consistent with A-B transition of the DNA. There is no detectable heat effect associated with this transition. At low water contents, the hydration of the DNACTA $(1: 1)$ complex is exothermic as for the pure DNA, but after incorporation of the first $7.0 \pm 0.1$ water molecules, the enthalpy changes sign. At 22 water molecules per base pair, the enthalpy levels off to $2.7 \pm 0.2 \mathrm{~kJ} / \mathrm{mol}$. In a separate experiment, the swelling limit for the DNACTA complex was found to be $27 \pm 1$ waters per base pair. The DNACTA complex is arranged in a hexagonal structure. We propose a model for the DNACTA complex based on the packing of the components in an electroneutral way consisting of six DNA helices, presumably in an A configuration, placed around a central $\mathrm{CTA}^{+}$cylinder. The hydration of the complex is seen as a balance between the attractive electrostatic interaction causing the formation of the complex and a repulsive component arising from a hexagonal deformation of $\mathrm{CTA}^{+}$cylinders. An important contribution to the partial molar enthalpy of water comes, in this interpretation, from the release of conformational constraints of the CTA ion alkyl chains.
\end{abstract}

\section{Introduction}

The interaction of cationic amphiphiles with DNA induces condensation of DNA into compact dense structures. ${ }^{1,2}$ Currently there is a considerable interest for such DNA-amphiphile complexes due to the possibility of applying such nonviral assemblies as vehicles for delivery of foreign DNA into cells. ${ }^{3,4}$ To control such a delivery process, it is essential to characterize the self-assembly processes, resulting in the formation of supramolecular structures. These structures contain substantial amounts of water, and the hydration of the charged DNA and lipid headgroups is an important factor in determining the properties of the complexes. Both lamellar and hexagonal structures have been found for stoichiometric aggregates of DNA and different lipids., ${ }^{1,2}$ Nearly all structural studies have been carried out on systems in equilibrium with a bulk aqueous solution of low osmotic pressure, and the extent, as well as the role of the hydration, have not been an issue. As the stoichiometric complex is formed in solution, counterions are released ${ }^{5}$ and the DNA-lipid aggregates are tightly packed with only a limited amount of water incorporated into the structure.

In addition to the importance for possible applications, the DNA-amphiphile complexes can also serve as useful model systems for studying the interaction between ionic groups in the presence of limited amounts of water. The precipitation of the anionic DNA-cationic amphiphile complex is due to an

* Author to whom correspondence may be addressed. E-mail: Cecilia.Leal@fkeml.lu.se.

$\dagger$ Physical Chemistry 1, Lund University.

$\doteqdot$ Building Materials, Lund University.

$\S$ University of Coimbra. attractive interaction that has both electrostatic and hydrophobic components. The fact that the precipitate contains 5-10 water molecules per charged group, when equilibrated relative to practically pure water, shows that repulsive interactions are also at play. In living systems, there are numerous examples of macromolecular assemblies where the components are separated by a thin aqueous layer. Currently, there is no established framework for a quantitative description of such a situation. One has a borderline case where theories based on a continuum description of the solvent break down, while there are still a large number of thermally excited molecular degrees of freedom. There is thus a case for collecting informative experimental data for systems complex enough to be interesting but still simple enough to allow for a detailed analysis.

In the present paper, we present a calorimetric study of the hydration of a stoichiometric complex between DNA and the cationic surfactant hexadecyltrimethylammonium bromide (CTAB). We have used a microcalorimetric vapor sorption technique developed by Wadsö and Markova ${ }^{6-8}$ that makes it possible to simultaneously measure both the partial molar free energy and the partial molar enthalpy of the water at nonsaturated conditions. Since corresponding data for the hydration of pure DNA is not available in the literature, we have, for reference purposes, performed measurements for this pure system as well.

\section{Materials and Methods}

Materials. We have used two types of sodium salt DNA, calf thymus DNA (fibrous type 1 "highly polymerized") and salmon testes DNA. They were both obtained from Sigma and 
used as received. All calf thymus DNA molecules are larger than $12 \mathrm{kbp}$; salmon testes DNA is highly polydisperse with an average molar mass of $2 \mathrm{kbp}$, as estimated by electrophoresis ( 0.8 wt $\%$ agarose gel in TBE). When DNA was purified by ethanol precipitation, no major differences were observed in the electrophoresis between purified and "as received" DNA. The concentration of DNA was determined by a spectroscopic method using the molar extinction coefficient $\epsilon_{260}=6600 \mathrm{~L}$ $\mathrm{mol}^{-1} \mathrm{~cm}^{-1}$ at $260 \mathrm{~nm}$. The $A_{260} / A_{280}$ ratio of DNA solutions was found to be 1.9, suggesting that DNA was free of proteins. ${ }^{9}$ CTAB (Merck p.a. quality) and sodium bromide (RiedeldeHaen extrapure quality) were used as received. The water used was from a Milli-Q filtration system (Millipore).

For the sorption microcalorimeter, an experiment with morphine sulfate (fine powder 99,6\% pure, Macfarlan Smith, Edinburgh, UK) was used to test the instrument. The results obtained agreed with published results. ${ }^{8}$ To avoid diffusion problems, it is important that the solid sample in the calorimeter is in a form of a fine powder. The present samples were powdery for the DNACTA complex and fibrous for DNA.

Sample Preparation. DNA solutions were prepared by weighing the desired amount and dissolving it in $10 \mathrm{mM} \mathrm{NaBr}$. The $\mathrm{pH}$ of all solutions was $7 \pm 0.2$. DNA-surfactant stoichiometric aggregates were prepared by mixing equimolar solutions $(7 \mathrm{mM})$ of DNA calf thymus and CTAB under stirring. The precipitate was equilibrated in solution for $48 \mathrm{~h}$. It was then separated from the aqueous phase by filtration (Duran, pore size $12 \mu \mathrm{m}$ ) at reduced pressure and washed extensively with Millipore water. The macromolecular complex salt (DNACTA) was dried for 3 days in a DW6-85 freeze-dryer. For sorption experiments, the samples were further dried in high vacuum during $24 \mathrm{~h}$ at room temperature and loaded at dry atmosphere inside a glovebox.

Sorption Microcalorimetry. A double-twin isothermal microcalorimeter was used to study the hydration of DNA and DNACTA complex. A detailed description of the instrument is given elsewhere. ${ }^{6-8}$ Several applications of this new technique have been carried out, such as studies of phospholipid hydration $^{10,11}$ and hydration of single-chain alkylglucosides. ${ }^{12}$

The instrument consists of a calorimetric cell constituted by two vessels connected by a stainless steel tube and an isothermal double-twin microcalorimeter placed in a thermostat. The cell is arranged so that the top vessel is in the top calorimeter and the bottom vessel is in the bottom calorimeter.

At the start of the measurement, the bottom vessel contains 20-100 mg of a dry sample and the top vessel about $100 \mu \mathrm{L}$ of water. During the measurement, water is vaporized and diffuses through the steel tube (the maximum diffusional flow is about $0.4 \mu \mathrm{g}$ of $\mathrm{H}_{2} \mathrm{O}$ per s) down to the bottom vessel where it is taken up by the sample. An experiment can be seen as a continuous titration of a dry sample with water vapor, and a measurement is stopped after 3-7 days when the water activity of the sample has reached 0.90-0.95. With this method, one can get both sorption isotherms (water content as a function of water activity) and the differential enthalpy of sorption.

The double microcalorimeter continuously and separately measures the thermal powers of the processes of vaporization $\left(P_{\mathrm{v}}\right)$ and sorption $\left(P_{\mathrm{s}}\right)$. The water flow rate, $q_{\mathrm{m}}(\mathrm{g} / \mathrm{s})$, from the top vessel to the sample vessel is calculated as

$$
q_{\mathrm{m}}=P_{\mathrm{v}} / \Delta H_{\mathrm{v}}
$$

where $\Delta H_{\mathrm{v}}(\mathrm{J} / \mathrm{g})$ is the enthalpy of vaporization of water. By use of Fick's law, one can calculate the diffusion of water vapor

$$
q_{\mathrm{m}}=k\left(p_{\mathrm{sat}}-p_{\mathrm{w}}\right)
$$

where $k$ is the diffusional permeability for water vapor through the gas phase between the top and the bottom vessels, $p_{\text {sat }}$ is the saturation vapor pressure of water, and $p_{\mathrm{w}}$ refers to the vapor pressure in the sample vessel. A calibration experiment with a drying agent such as molecular sieves acting as sample (with a water vapor pressure close to zero) is made to find the maximal mass flow rate $q_{\mathrm{m}, \mathrm{max}}$

$$
q_{\mathrm{m}}=q_{\mathrm{m}, \max }=k p_{\mathrm{sat}}
$$

By combination of eqs 2 and 3, one can get the water activity (for practical purposes here defined as $P_{\mathrm{w}} / p_{\mathrm{sat}}$ )

$$
a_{\mathrm{w}}=1-q_{\mathrm{m}} / q_{\mathrm{m}, \max }
$$

The water gain of the sample, $C_{\mathrm{w}}(\mathrm{g} / \mathrm{g})$, is

$$
C_{\mathrm{w}}=\frac{1}{m_{0}} \int\left(q_{\mathrm{m}} \mathrm{d} t\right)
$$

where $m_{0}$ is the mass of the sample in the beginning of the measurement. In the experiments, the differential enthalpy of sorption, $\Delta H_{\text {sorp }}$, corresponds to the enthalpy change as a small amount of liquid water at atmospheric pressure is added to the sample, and it is calculated relative to the liquid state of water as

$$
\Delta H_{\text {sorp }}=\frac{\left(P_{\mathrm{s}}-P_{\mathrm{v}}\right)}{q_{\mathrm{m}}}
$$

Provided equilibrium is maintained, $\Delta H_{\text {sorp }}$ corresponds to the difference between the partial enthalpy of the water $\left(\Delta \bar{H}_{\mathrm{w}}\right)$ in the sample and in liquid water.

\section{Results}

DNA Hydration. Sorption Isotherm. The calorimetric sorption isotherm gives a relation between the water content in the sample and the chemical potential of the water expressed in terms of the activity (or equivalent relative vapor pressure). Figure 1 shows the results for DNA at different initial masses. Two of the sorption experiments (51.1 mg calf thymus and 44.1 salmon testes) reached 0.84 water activity, while others were conducted for longer periods of time reaching 0.92 water activity (44.5 $\mathrm{mg}$ and $21.3 \mathrm{mg}$ calf thymus). Before the experiments, the samples were dried under high vacuum for $24 \mathrm{~h}$. At the start of the experiments, the water activity was measured to be 0.02 , indicating that the samples were not totally dry. It has been shown that some water molecules per base pair may remain even in extensively dried samples. ${ }^{13}$ This introduces a small uncertainty in the absolute values of the water content but will not influence the features of the isotherm at higher water activities, which are at the focus of interest.

The sorption curves for different initial masses of DNA show similar features, but they are slightly shifted relative to each other at low water contents, probably reflecting the difficulty in achieving an equilibrium structure under very dry conditions. In Figure 1 is also shown the early results by Falk et al. ${ }^{14}$ measured gravimetrically. There is a full agreement between the two methods within the experimental accuracy. This additionally indicates that, if our "dry" samples contain some water, we also quantitatively reproduce this feature of the older study. 


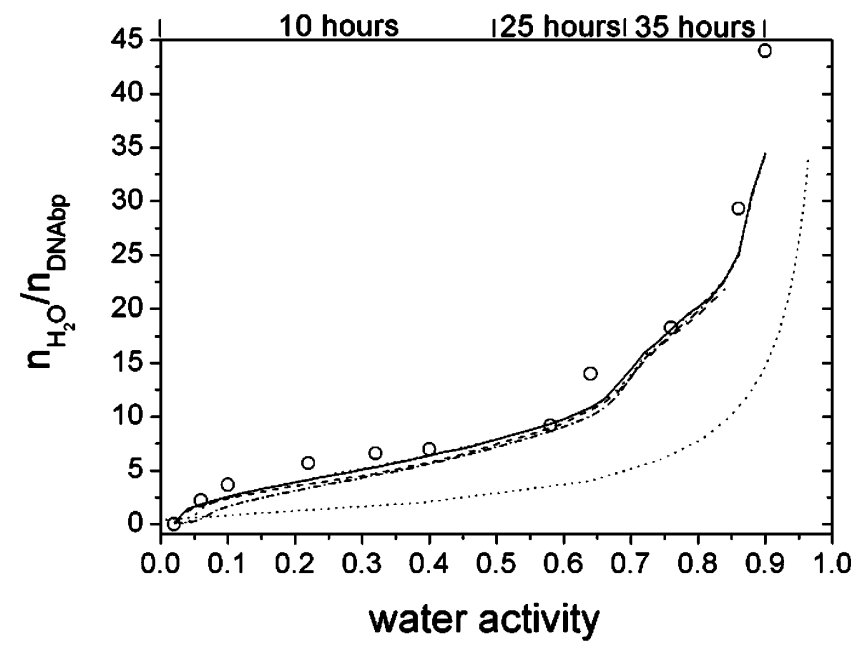

Figure 1. Sorption isotherm for DNA of different sources and initial masses ((solid line) $44.5 \mathrm{mg}$ calf thymus, (dashed line) $51.2 \mathrm{mg}$ calf thymus, (dot-dashed line) $21.3 \mathrm{mg}$ calf thymus, (dotted line) $44.1 \mathrm{mg}$ salmon testes): water content (water molecules/DNA base pair) vs water activity. The dotted curve corresponds to water content as a function of water activity calculated from the Poisson-Boltzmann equation. The open circles correspond to the gravimetric data for NaDNA calf thymus hydration obtained by Falk et al. ${ }^{14}$ Time involved in the sorption process is showed at the top of the graph.

The sorption experiments are rather slow; in the first $10 \mathrm{~h}$, we reach 0.5 water activity, while $60 \mathrm{~h}$ more are necessary to reach 0.9 water activity. Toward the end of the experiments, the diffusional transport process becomes very slow and the heat effects are lost in the noise. This puts an upper limit to the accessible range of water activity.

We can observe different regimes in the sorption isotherm for DNA. The water gain gradually rises up to $10.0 \pm 0.4$ water molecules per DNA base pair and 0.62 water activity, at this point we observe a kink in the isotherm. The exact location of the kinks was obtained by the differentiated isotherms not presented here. When the water activity is 0.80 and the water molecules per base pair are about $20.0 \pm 0.3$, another kink in the curve is observed. The experiment ends at 0.92 water activity, and DNA takes up $35.0 \pm 0.1$ water molecules per base pair.

Sorption Enthalpies. The novel aspect of the present technique is that the partial molar enthalpy of water is obtained simultaneously with the partial molar free energy (sorption isotherms). Figure 2a shows the partial molar enthalpy of the water $\left(\Delta \bar{H}_{\mathrm{w}}\right)$ plotted against the water content. Arrows in the graph correspond to the kinks in the isotherm. At low water contents, the enthalpy is strongly negative and it increases gradually with increasing water content. At approximately 20 water molecules per base pair, the enthalpy has increased to the level where the difference from zero is smaller than the experimental uncertainty $(0.5-1 \mathrm{~kJ} / \mathrm{mol})$. We note that there are no significant changes in the enthalpy values at the observed kinks in the isotherm. Figure $2 \mathrm{~b}$ shows the calculated integral of the partial molar enthalpy (differential enthalpy of sorption).

DNACTA Hydration. Sorption Isotherms. In Figure 3, the sorption isotherms for different masses of DNACTA complexes are presented. The curves are similar, suggesting that the sorption process takes place under quasiequilibrium conditions. One of the experiments $(48.6 \mathrm{mg}$ ) was conducted for 5 days until 0.92 water activity was reached, while the other $(25.0 \mathrm{mg})$ was carried out for 7 days until 0.96 water activity. As the water activity has increased to 0.68 , not more than $7.0 \pm 0.1$ water molecules are absorbed by the complex. After this point, the

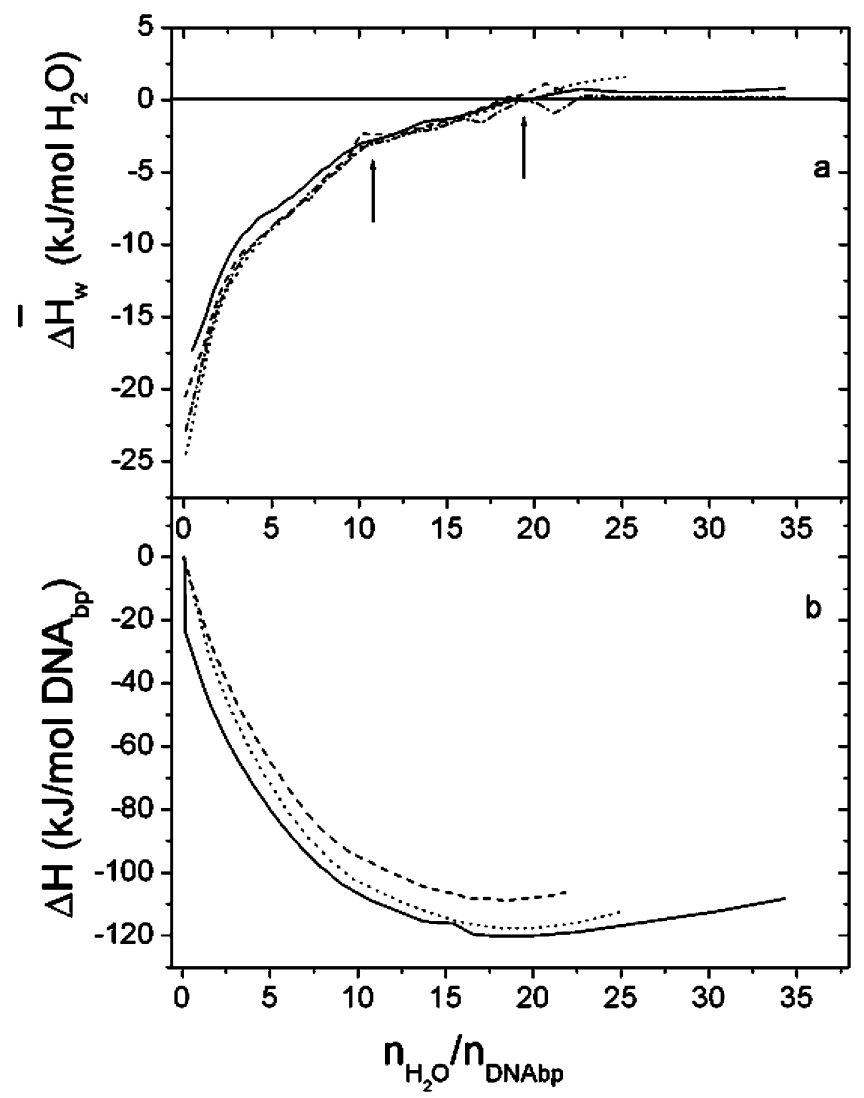

Figure 2. (a) Partial molar enthalpy of water in the sorption process $\left(\Delta \bar{H}_{\mathrm{w}}\right)$ as a function of water content. Arrows indicate the point where isotherm kinks appear. (b) Calculated integral of partial molar enthalpy of water $(\Delta H)$ vs water content ((solid line) $44.5 \mathrm{mg}$ calf thymus, (dashed line) $51.2 \mathrm{mg}$ calf thymus, (dot-dashed line) $21.3 \mathrm{mg}$ calf thymus, (dotted line) $44.1 \mathrm{mg}$ salmon testes).

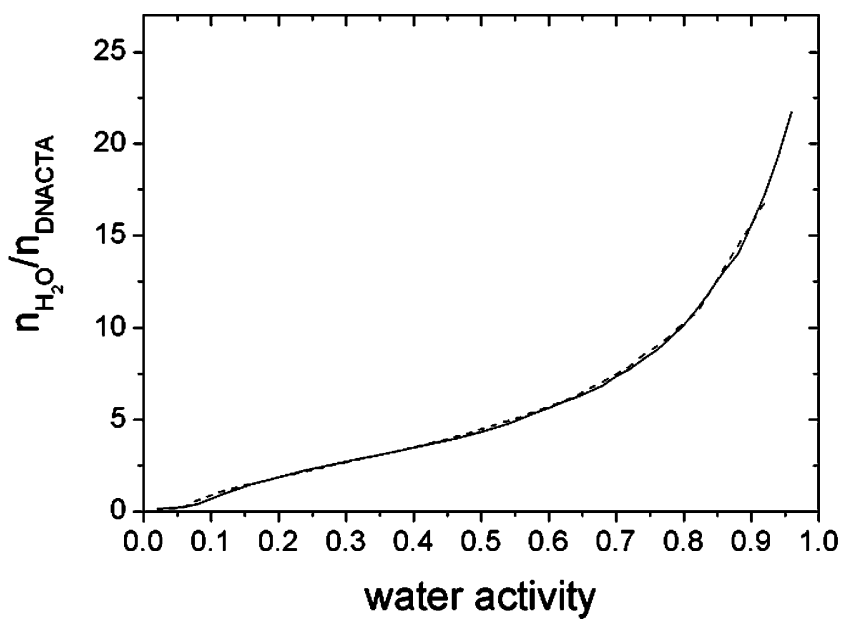

Figure 3. Sorption isotherm at $25^{\circ} \mathrm{C}$ for DNACTA ((solid line) 25.0 $\mathrm{mg}$; (dashed line) $48.6 \mathrm{mg}$ ): water content (water molecules/DNACTA) vs water activity.

absorbed amount increases more pronouncedly, and at 0.92 water activity, $17.0 \pm 0.3$ water molecules are incorporated in the complex. For the $25 \mathrm{mg}$ DNACTA sample, 22 water molecules are incorporated at 0.96 water activity. No kinks or plateaus are observed in the curves. In an independent sorption experiment, where samples were allowed to equilibrate for a long time in a desiccator with a salt solution of relative humidity of $99.5 \%$, the water uptake was measured as $27 \pm 1$ water molecules per base pair, which in practice should correspond to the swelling limit. 


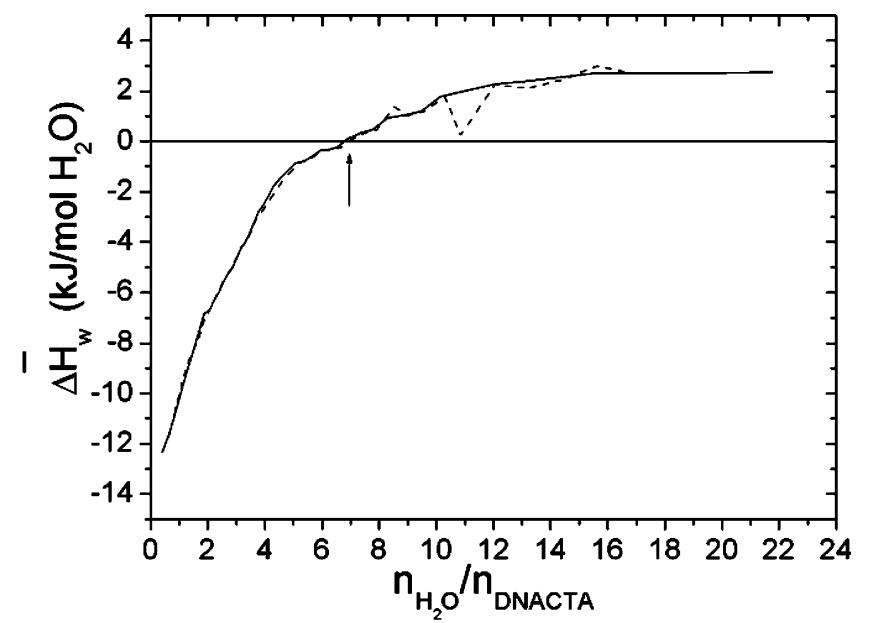

Figure 4. Partial molar enthalpy of water in the sorption process $\left(\Delta \bar{H}_{\mathrm{w}}\right)$ as a function of water content for DNACTA ((solid line) $25.0 \mathrm{mg}$; (dashed line) $48.6 \mathrm{mg}$ ).

Sorption Enthalpies. In Figure 4, we present the partial molar enthalpy vs the amount of water. The enthalpy is strongly negative at low water contents. As for the pure DNA, it increases monotonically with increasing water content, but at the point indicated by the arrow in the graph, the enthalpy goes through zero. This occurs at $7.0 \pm 0.1$ water molecules per base pair, and it corresponds to a water activity of 0.68 in the sorption isotherm. The enthalpy continues to increase until the complex has absorbed approximately 12 water molecules. A further increase in the water content leads to an apparently constant value of the partial molar enthalpy of $2.7 \pm 0.2 \mathrm{~kJ} / \mathrm{mol} \mathrm{H}_{2} \mathrm{O}$.

\section{Discussion}

DNA Hydration. Although the main focus of this investigation is on the DNA-surfactant complex, measurements were performed on pure DNA-water systems to provide reference data. DNA hydration has been extensively studied by several gravimetric, spectroscopic, osmotic stress, calorimetric, and scattering methods. ${ }^{14-24}$ However, to our knowledge, simultaneous free energy and enthalpy data have not been reported previously.

It is well established that, by reducing the vapor pressure, one can induce a transition from the common B form of DNA to the more compact A form. This occurs at a water activity of approximately 0.80 , and there are around 20 water molecules per base pair at the transition. ${ }^{9}$ In the sorption isotherm presented in Figure 1, the kink at 0.8 water activity may be attributed to the well-known $\mathrm{A}-\mathrm{B}$ transition. In a pure homogeneous system, an A to B transition should have a first-order character with a jump in water content at constant water activity. However, in practice, inhomogeneities and kinetic effects lead to a certain smearing of the transition and one only observes a steep rise in water content signaled by the kink in the curve. We do not observe any strong enthalpy effects at this transition although it is associated with substantial conformational changes in the DNA molecule.

With monovalent counterions in water, DNA behaves as a typical polyelectrolyte and swells indefinitely if exposed to water vapor at saturation pressure. At long range, the DNA-DNA interaction is dominated by the electrostatic double-layer forces, while deviations from the continuum electrostatic description are expected for more concentrated systems. However, experience has shown that a description based on the Poisson-

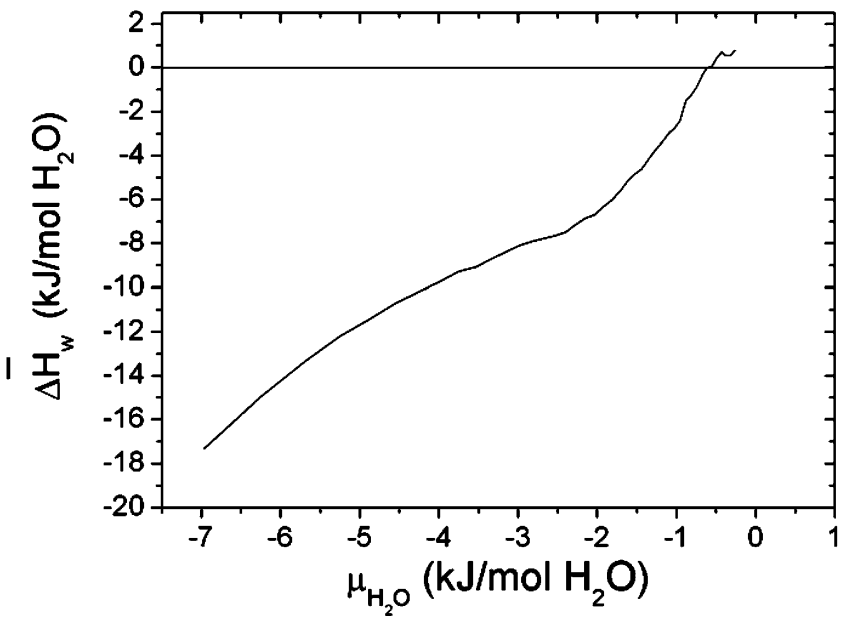

Figure 5. Partial molar enthalpy of water $\left(\Delta \bar{H}_{w}\right)$ vs partial molar free energy of water $\left(\mu_{\mathrm{H}_{2} \mathrm{O}}\right)$ for DNA (21.3 mg calf thymus).

Boltzmann theory can give reasonable results even for very dense systems. ${ }^{25}$ It is thus informative to work out the predictions of the PB theory for the pure DNA system by applying a cell model, ${ }^{26}$ with DNA represented as a smooth uniformly charged cylinder $\left(2 \mathrm{e}^{-} / 3.46 \AA\right)$, of radius $11 \AA$ in the $\mathrm{B}$ form, located in a cylindrical cell with an outer radius determined by the water content. In the experiments, the electrolyte concentration is negligible relative to the counterions, so one use the counterion-only solution. ${ }^{27}$

In Figure 1, we show the sorption isotherm calculated from the PB equation. It is clear that the PB data set (dotted curve) falls below the experimental isotherm, but although the discrepancy is substantial, the calculations do give the right order of magnitude. In this situation with limited amounts of water available, it becomes an essential complication that the DNA double helix has a molecularly rough surface with grooves so that the uniform cylinder approximation becomes problematic. At higher water contents, the degrees of freedom of the polymer backbone also contributes to reduce the water activity. The PB description not only gives a prediction of the osmotic pressure through the counterion concentration on the outer cell boundary, but it also provides the electrostatic "energy" through an integration of the electric field squared. This "energy" is from a thermodynamic point of view a free energy, since it contains a temperature-dependent dielectric constant. ${ }^{28}$ To obtain a molecular understanding of the electrostatic contribution to the partial molar enthalpy of water, one can start by realizing that adding water to a DNA system results in a separation of charges on average. The integral of the field squared increases, indicating the enthalpy should be positive. However, due to the unusual temperature dependence of the dielectric constant for water, the proper thermodynamic quantity has the opposite sign, giving a negative contribution to the partial molar enthalpy. Furthermore, the continuum picture of the water-charge interaction is problematic at these short distances.

For the pure DNA system, the partial molar enthalpy is negative over the entire range it is measured. Also, in this case, the electrostatic model predicts qualitatively the correct sign of the effect, but for the enthalpy, the quantitative discrepancy is larger. In Figure 5, the partial molar enthalpy is plotted vs the partial molar free energy, and the $\Delta \bar{H}_{\mathrm{w}}$ values are consistently larger than the $\mu_{\mathrm{H}_{2} \mathrm{O}}$ values by approximately a factor of 2 . This means then that the entropy term $-T S$ is positive but of the same order of magnitude as the partial molar free energy. In addition to the ionic species, there are a number of polar groups 
on the surface of the DNA, and the hydration of these groups contributes to both free energy and enthalpy at low water contents.

DNACTA Hydration. Sorption Isotherm. We have prepared stoichiometric complexes of DNA and a cationic surfactant, CTAB, where DNA counterions $\left(\mathrm{Na}^{+}\right)$were replaced by the surfactant cation $\left(\mathrm{CTA}^{+}\right)$. This complex (DNACTA) is a precipitate that can be separated from the aqueous phase by filtration and washed with pure water. At charge neutralization, counterion release is maximal and nearly complete. ${ }^{5}$ Before the sorption experiments, we dried the samples to remove water incorporated within the DNACTA complex. During the experiment, water molecules are incorporated back in the complex, and the energy involved with that process is measured.

According to current models, DNA-amphiphile aggregates are tightly packed ${ }^{1}$ and the amount of water entrapped within them should be rather limited. From the sorption isotherm, we see that, when the water activity is around 0.96, the complex contains 22 water molecules per base pair and desiccator experiments yield a value of 27 water molecules per base pair in the limit of a water activity of 1 . It is clear that the precipitation of the complex, and thus its finite swelling in water, is due to an electrostatic attraction between the positive amphiphile and the negative DNA. May et al. ${ }^{29}$ have presented a quantitative model for the electrostatic interaction in this type of system.

It is more problematic to find the cause of the swelling leading to the incorporation of up to 27 water molecules per base pair. It is a common observation that systems consisting of a polyelectrolyte and an oppositely charged amphiphile form aggregates with a finite water content. ${ }^{30-32}$ A general interpretation of this finding is that it is difficult to obtain a locally perfect charge matching.

That there is any water uptake at all in the presently studied system can be qualitatively attributed to the fact that both DNA and the surfactant have preferred aggregate geometries, and a perfect charge-charge matching at short range is not optimal. During the swelling, DNA apparently does not change conformation cooperatively, as we do not observe any kink in the sorption curve when hydrating the complex again. Infrared studies of DNA - lipid aggregates show that the conformational state of DNA after condensation with amphiphiles in aqueous solution might differ from the native $\mathrm{B}$ form present in solution. $\mathrm{A}, \mathrm{B}$, and $\mathrm{C}$ forms of DNA have been suggested as well as mixtures of different DNA forms. ${ }^{33}$ For certain DNA-CTAB systems, circular dichroism experiments have shown that the DNA remains essentially in B form as CTAB binds to the duplex. However, in these studied complexes, DNA is in excess relative to $\mathrm{CTAB}$ since one is below the precipitation limit ${ }^{34}$ or the concentrations are low $^{35}$ such that the binding degree of CTAB to DNA is only $60 \% .{ }^{36}$ In our studies, the DNACTA complex is a neutral precipitate. We consider it an open question what conformation the DNA takes in the precipitate.

Sorption Enthalpies. In the DNACTA system, there is a tight packing of DNA helices and surfactant aggregates, and the sodium counterions of the pure DNA system are replaced by the more bulky quaternary ammonium groups. For lower water contents, the partial molar enthalpy of the water is at least a factor of 2 less negative than for the pure DNA system. Since the charged groups have the strongest interactions with water, they are the first to be hydrated; we interpret the observed difference at low water contents as mainly reflecting the difference between hydration of sodium ions relative to quaternary ammonium ions. A second significant difference is that

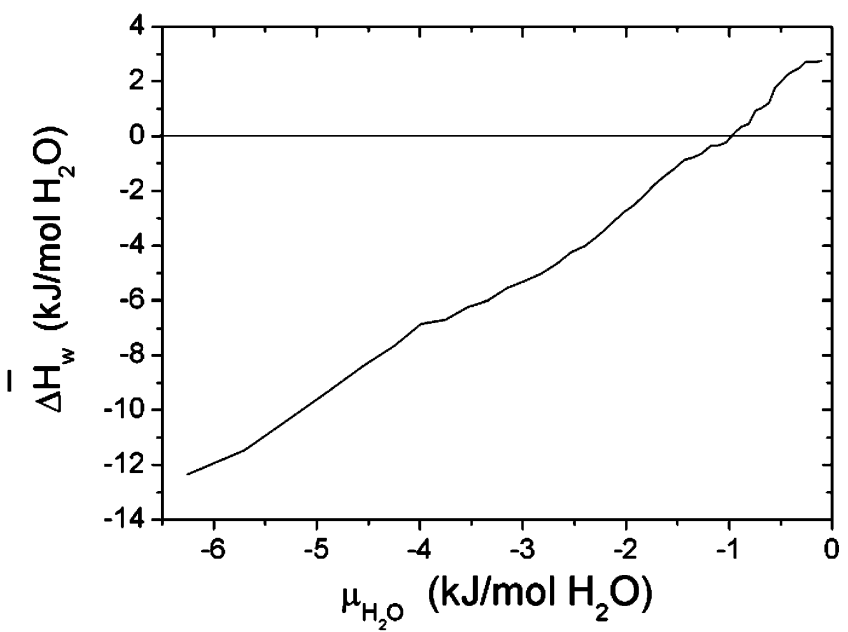

Figure 6. Partial molar enthalpy of water $\left(\Delta \bar{H}_{\mathrm{w}}\right)$ versus partial molar free energy of water $\left(\mu_{\mathrm{H}_{2} \mathrm{O}}\right)$ for DNACTA $(25.0 \mathrm{mg})$.

for the DNACTA case, enthalpy values turn positive after seven water molecules per base pair have been added. From a thermodynamic perspective, this means that, between 7 and 25 water molecules per base pair, the sorption is driven by an entropic rather than an enthalpic effect. Further insight into this balance between entropy and enthalpy is obtained from Figure 6 , which shows that there is, in a way analogous to found in Figure 5, an approximately linear relation between the partial molar free energy and enthalpy, but in this case with a nonzero intercept.

Mechanism of Swelling and the Structure of the DNASurfactant Complex. The stoichiometric DNACTAB (1:1) precipitate has previously been studied by X-ray (small- and wide-angle) scattering. ${ }^{2}$ We repeated such experiments on samples prepared the same way as for the calorimetric measurements and found agreement with the results of Mel'nikov et al. The small-angle region showed 2 diffraction peaks consistent with a $2 \mathrm{D}$ hexagonal lattice with a period of $49.4 \AA$ for the DNACTA complex prepared at water activity of 1.0. In the wide-angle region, no peak was detected. We performed ${ }^{1} \mathrm{H}$ NMR on dry and wet DNACTA precipitates, ${ }^{37}$ and for the fully hydrated complex, several narrow peaks were observed, whereas for the dry complex there was one rather broad peak. The spectrum for the wet complex is similar to the one observed for CTAB rodlike micelles arranged in an hexagonal structure. ${ }^{38}$ This indicates that, in the most hydrated state of the DNACTA complex, the amphiphile is arranged as in a liquid crystalline hexagonal structure.

When DNA interacts with double-chain lipids that by themselves form lamellar liquid crystals, the DNA-lipid complex is also lamellar. ${ }^{1}$ Thus, it is a reasonable assumption that, since CTAB by itself easily forms a normal hexagonal phase, the structure of the hexagonal DNACTA complex is also of the normal type. The fact that there are no extra counterions implies that the DNA and the CTA must pack in such a way that there is charge neutralization on as small a scale as possible. In the hexagonal phase of $\mathrm{CTAB}$, there is an area per headgroup of $53 \AA^{2}$ and a cylinder diameter of $43 \AA .{ }^{39}$ This means that, projected on the cylinder axis, there is one charge per $0.36 \AA$. Surfactant aggregates are formed by a self-assembly process so that under different conditions one can expect adjustments in these numbers, but there should not occur major changes; a change in headgroup area exceeding, for example, $20 \%$ appears unlikely. For DNA, there are more fixed atomic positions in the double helix so the structure is locally stiffer. On the other 
hand, there are different conformations of the helix, and for the pure DNA in particular, we considered the $\mathrm{A}-\mathrm{B}$ transition. The general observation is that A DNA is favored when the electrostatic repulsion along the helix is reduced either by lowering the vapor pressure or by the addition of salt. ${ }^{9}$ Binding to the highly positive CTA aggregate is an extreme case of reducing the electrostatic repulsion, and on the basis of this argument, one should expect that in the DNACTA system the A form is favored relative to the B one. However, for systems where DNA is in excess relative to the surfactant, there are spectroscopic CD observations that DNA remains in the B form on complexation. ${ }^{34,35}$ At present, this issue appears unresolved, but it has a significant bearing on the possible mutual arrangement of DNA helices and CTA cylinders in the complex.

In the A form, DNA has approximately one unit charge per $1.27 \AA$, and in the $\mathrm{B}$ form, it has one unit charge per $1.72 \AA$ when projected onto the axis. If one does not allow for structural rearrangements, this would imply a ratio of 3:5 DNA helices per CTA cylinder with A DNA and a ratio of $4: 8$ with B DNA. These numbers, combined with the measured lattice spacing and the estimated cylinder radii, put rather strict conditions on conceivable structures of the hexagonal packing. The radius of the CTA cylinder is approximately twice that of the DNA and it is reasonable to place it in the center of the hexagonal cell. With a 6-fold symmetry, there are essentially three ways to place the DNA helices around the central CTA cylinder that could be consistent with the charge neutralization condition. These are illustrated in Figure 7. The possibility a gives a 2:1 ratio, which is clearly too small to give charge neutralization unless there are major structural changes of the DNA and/or CTA cylinders. The alternative $b$ with a ratio of $3: 1$ could be compatible with charge neutralization if the DNA is in the A form, while the possibility $\mathrm{c}$, which is a combination of a and $\mathrm{b}$, gives a ratio of 5:1, which fits if DNA is in the B form. However, in this structure, there are 12 DNA double helices spread around the periphery of one CTA cylinder, and with a helix radius of $10-11 \AA$, there is hardly space for these cylinders, given a spacing of $49 \AA$ between the (1 0$)$ planes. (See caption in Figure 7). Thus, from a consideration of the possibilities of packing the components in an electroneutral way, we arrive at the conclusion that the basic structure shown in Figure $7 \mathrm{~b}$ is the most likely one.

In such a structure, adjustments must occur to satisfy the neutrality condition. This can be accomplished by either expanding the CTA cylinders somewhat in the longitudinal direction and/or by slightly twisting the DNA helices around the central cylinder. Whichever occurs, there is also another type of adjustment in response to obtaining optimal packing from an interaction point of view. With the countercharge localized to six helices, the surfactant aggregate senses a pronounced 6-fold local symmetry and its cross sectional area should reflect this deviation from lateral isotropy. Hence one could write in plane the contour of the aggregate, $R$, as

$$
R=R_{0}(1+a \sin (6 \Phi))
$$

where $R_{0}$ is the radius of a cylinder and the parameter $a$ measures the deviation from cylindrical symmetry. In Figure 8 we show typical expected packing.

Such a model provides a basis for a discussion of the hydration behavior. The space outside the surfactant aggregates and the DNA helices is occupied with water. Thus, the lower the water content, the larger we expect the deviation parameter $a$ to be and one can see the driving force for the swelling as a)

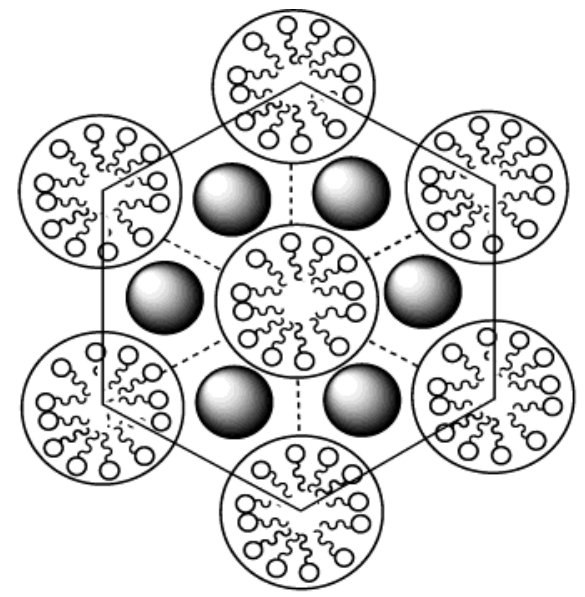

b)

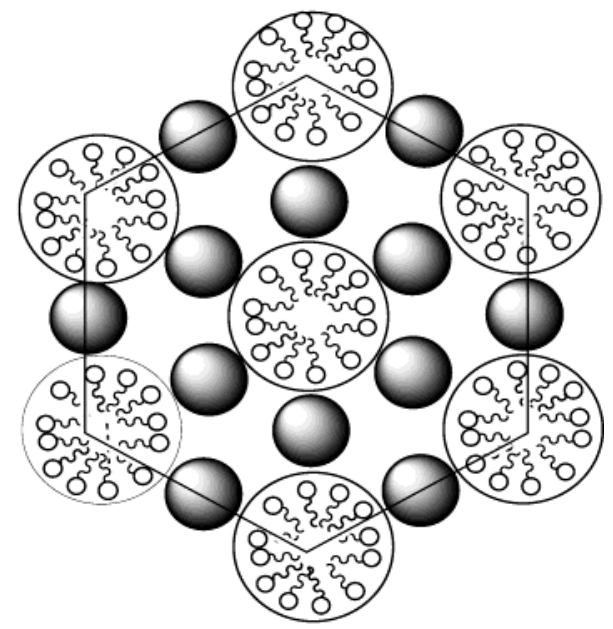

c)

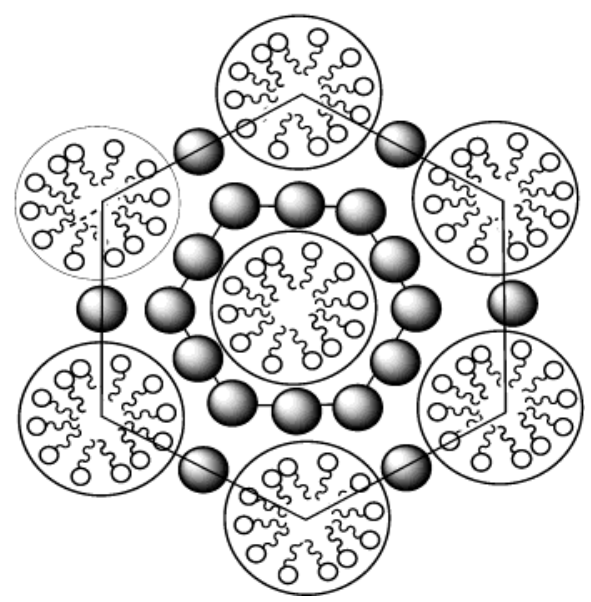

Figure 7. Illustration of different packing arrangements for an electroneutral DNACTA complex consisting of a 6-fold symmetry of DNA helices (darker circles) around the central CTA cylinder for different ratios of (DNA helices/CTA cylinder): (a) 2:1, (b) 3:1, and (c) 5:1. Considering the CTA radius $21.5 \AA$ and the DNA radius $10 \AA$, the cross-sectional areas occupied in the cells are: (a) $62 \mathrm{~nm}^{2}$, (b) 72 $\mathrm{nm}^{2}$, and (c) $90 \mathrm{~nm}^{2}$. A (1 0 ) lattice spacing of $49 \AA$ corresponds to a total area of $83 \mathrm{~nm}^{2}$, making alternative c difficult to realize physically.

partly caused by a desire to reduce the deformation of the surfactant aggregate toward the intrinsically most favorable value of zero. By use of this perspective, the enthalpy data for the DNACTA complex can be interpreted in terms of a primary hydration of the charged groups, primarily the phosphates, involving approximately 7 water molecules per base pair. Subsequent addition of water up to the swelling limit involves primarily a decrease in cylinder deformation. This involves a 


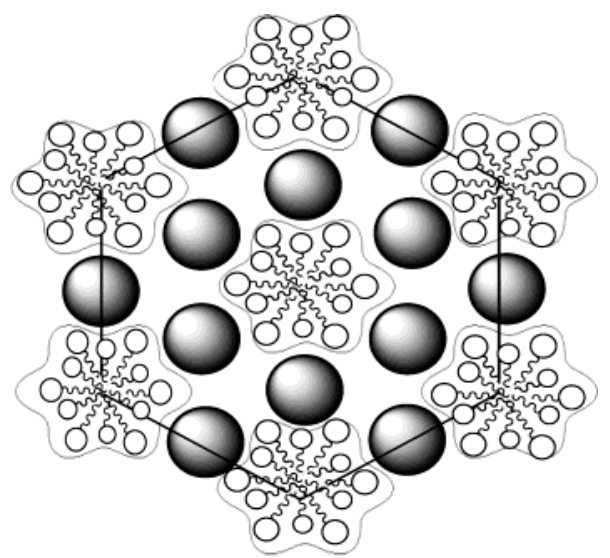

Figure 8. Expected DNACTA packing for an electroneutral complex consisting of a hexagonal arrangement of DNA helices (darker circles) around the central CTA distorted cylinder. The contour of the CTA cylinders consistent with eq 7 .

release of constraints on conformations of the surfactant chains, leading to an increase in entropy and enthalpy.

\section{Conclusions}

We present the first simultaneous measurements of enthalpy and free energy of hydration for pure DNA and for an amphiphile-DNA complex. For DNA, the hydration is exothermic at least up to 20 water molecules per base pair. The partial molar enthalpy and partial molar free energy are approximately proportional, with a slope of 2.5. The PoissonBoltzmann description of the electrostatic interactions gives the right composition dependence of water chemical potential but underestimates its magnitude. This model also predicts the right qualitative behavior of the enthalpies but quantitatively underestimates the effect substantially.

At full hydration of the DNACTA complex, i.e., when the complex is in equilibrium with pure water, there are $27 \pm 1$ water molecules per base pair. During the swelling, the water uptake in the complex is consistently below that observed for pure DNA. Also for the DNACTA complex, we find an approximately linear relation between enthalpy and free energy, but with a nonzero intercept. The enthalpy becomes positive around seven water molecules per base pair with a limiting value at full hydration of $2.7 \pm 0.2 \mathrm{~kJ} / \mathrm{mol}$. The structure of the DNACTA is discussed, and on the basis of a combination of the thermodynamic, structural small-angle X-ray, NMR data, and electroneutrality constraints, a tentative model of the DNACTA system is proposed. In the unit cell of the 2D hexagonal structure, there is a central (near) cylindrical CTA aggregate that is surrounded by six DNA helices. The CTA aggregate is hexagonally deformed, and this deformation increases with decreasing water content. Further work is in progress to clearly establish these structural aspects.

Acknowledgment. This work was supported by the Swedish Research Council for Engineering Sciences and the Fundação para a Ciência e Tecnologia (project Sapiens PCTI/99/QUI/ 35415).

\section{References and Notes}

(1) Koltover, I.; Salditt, T.; Rädler, J. O.; Safinya, C. R. Science 1998, 281,78 .

(2) Mel'nikov, S. M.; Sergeyev, V. G.; Yoshikawa, K.; Takahashi, H.; Hatta, I. J. Chem. Phys. 1997, 107, 6917.

(3) Felgner, P. L.; Gadek, T. R.; Holm, M.; Roman, R.; Chan, H. W.; Wenz, M.; Northrop, J. P.; Ringold, G. M.; Danielsen, M. Proc. Natl. Acad. Sci. U. S. A. 1987, 84, 7413.

(4) Felgner, P. L. Adv. Drug. Delivery Res. 1990, 5, 163.

(5) Wagner, K.; Harries, D.; May, S.; Kahl, V.; Rädler, J. O.; BenShaul, A. Langmuir 2000, 16, 303.

(6) Wadsö, L.; Markova, N. Thermochim. Acta 2000, 360, 101.

(7) Wadsö, L.; Markova, N. Rev. Sci. Instrum. 2002, 73, 2743.

(8) Wadsö, L.; Markova, N. Eur. J. Pharm. Biopharm. 2001, 51, 77.

(9) Saenger, W. Principles of Nucleic Acid Structure; SpringerVerlag: New York, 1984.

(10) Markova, N.; Sparr, E.; Wadsö, L.; Wennerstrom, H. J. Phys. Chem. B 2000, 104, 8053 .

93.

(11) Markova, N.; Sparr, E.; Wadsö, L. Thermochim. Acta 2001, 374,

(12) Kocherbitov, V.; Söderman, O.; Wadsö, L. J. Phys. Chem. B 2002. 106, 2910.

(13) Tao, N. J.; Lindsay, S. M. Biopolymers 1989, 28, 1019.

(14) Falk, M.; Hartman, K. A.; Lord, R. C. J. Am. Chem. Soc. 1962, $84,3843$.

(15) Lavalle, N.; Lee, S. A.; Rupprecht, A. Biopolymers 1990, 30, 877.

(16) Rau, D. C.; Lee, B.; Parsegian, A. Proc. Natl. Acad. Sci. U. S. A. 1984, 81, 2621

(17) Rau, D. C.; Parsegian, V. A. Biophys. J. 1992, 61, 246.

(18) Mrevlishvili, G. M.; Carvalho, A. P. S. M. C.; Ribeiro da Silva, M. A. V.; Mdzinarashvili, T. D.; Razmadze, G. Z.; Tarielashvili, T. Z. J. Therm. Anal. Cal. 2001, 66, 133 .

(19) Mrevlishvili, G. M.; Carvalho, A. P. S. M. C.; Ribeiro da Silva, M. A. V. Thermochim. Acta 2002, 394, 73.

(20) Egli, M.; Tereshko, M.; Teplova, M.; Minasov, G.; Joachimiak, A.; Sanishvili, R.; Weeks, C. M.; Miller, R.; Maier, H.; An, H.; Cook, P. Dan; Manoharan, M. Biopolymers 1998, 48, 234.

(21) Dickerson, R. E. J. Mol. Biol. 1983, 166, 419.

(22) Feig, M.; Pettitt, B. M. Biopolymers 1998, 48, 199.

(23) Schneider, B.; Patel, K.; Berman, H. M. Biophys. J. 1998, 75, 2422.

(24) Texter, J. Prog. Biophys. Mol. Biol. 1978, 33, 83.

(25) Jönsson, B.; Wennerstrom, H. J. Phys. Chem. 1987, 91, 338.

(26) Wennerström, H.; Jönsson, B.; Linse, P. J. Chem. Phys. 1982, 76, 4665 .

(27) Fuoss, R. M.; Katchalsky A.; Lifson, S. Proc. Natl. Acad. Sci. U. S. A. 1951, 37, 579 .

(28) Evans, D. F.; Wennerström, H. The Colloidal Domain: Where Physics, Biology and Technology Meet; VCH: New York, 1998.

(29) May, S.; Harries, D.; Ben-Shaul, A. Biophys. J. 2000, 78, 1681.

(30) Piculell, L.; Lindman, B. Adv. Colloid Interface Sci. 1992, 41, 149.

(31) Svensson, A.; Piculell, L.; Cabane, B.; Ilekti, P. J. Phys. Chem. B. 2002, 106, 1013.

(32) Thalberg, K.; Lindman, B. Polymer-Surfactant Interactions-Recent Developments. In Interactions of Surfactants with Polymers and Proteins; Goddard, E. D., Ananthapadmanabhan, K. P., Eds.; CRC Press: Boca Raton, FL, 1993; p 203.

(33) Pohle, W.; Selle, C.; Gauger, D. R.; Zantl, R.; Artzner, F.; Raidler, J. O. Phys. Chem. Chem. Phys. 2000, 2, 4642.

(34) Spink C. H.; Chaires J. B. J. Am. Chem. Soc. 1997, 119, 10920.

(35) Ghirlando, R.; Wachtel, J.; Arad, T.; Minsky, A. Biochemistry 1992, 31,7110 .

(36) Mel'nikov, S. M.; Sergeyev, V. G.; Yoshikawa, K. J. Am. Chem. Soc. 1995, 117, 9951.

(37) Leal, C.; Topgaard, D.; Wennerström, H. To be published.

(38) Ulmius, J.; Wennerström, H. J. Magn. Reson. 1977, 28, 309.

(39) Fontell, K.; Khan, A.; Lindström, B.; Maciejewska, D.; Puang-

Ngern, S. Colloid Polym. Sci. 1991, 269, 727. 